Homology, Homotopy and Applications, vol.16(1), 2014, pp.139-157

\title{
POSTNIKOV TOWERS WITH FIBERS GENERALIZED EILENBERG-MAC LANE SPACES
}

\author{
KOUYEMON IRIYE AND DAISUKE KISHIMOTO
}

\author{
(communicated by Ronald Brown)
}

\begin{abstract}
A generalized Postnikov tower (GPT) is defined as a tower of principal fibrations with the classifying maps into generalized Eilenberg-Mac Lane spaces. We study fundamental properties of GPT's such as their existence, localization and length. We further consider the distribution of torsion in a GPT of a finite complex, motivated by the result of McGibbon and Neisendorfer $[\mathbf{M N}]$. We also give an algebraic description of the length of a GPT of a rational space.
\end{abstract}

\section{Introduction}

Decomposing a space into a tower of fibrations is a standard technique in homotopy theory. One readily conceives the Postnikov tower as an example of such a decomposition. Recall that the Postnikov tower of a path-connected space $X$ is a tower of fibrations

$$
\cdots \rightarrow X_{n} \rightarrow X_{n-1} \rightarrow \cdots \rightarrow X_{0}=*
$$

such that the fiber of $X_{n} \rightarrow X_{n-1}$ is $K\left(\pi_{n}(X), n\right)$ for each $n$ and that there is a weak homotopy equivalence $X_{n} \rightarrow \lim X_{n}$. Although the Postnikov tower is one of the most useful decompositions of spaces, it sometimes seems redundant. For example, the length of the Postnikov tower of a product of Eilenberg-Mac Lane spaces can be infinite, but each fibration in the tower is trivial. So one might naively want to bundle all of these trivial fibrations. This is, of course, caused by the dimensional constraint; i.e., the fiber of $X_{n} \rightarrow X_{n-1}$ is $K\left(\pi_{n}(X), n\right)$. Relaxing this constraint, certain generalizations of Postnikov towers were introduced in [GM, Ma, Sm]. More general towers were also introduced in $[\mathbf{F}]$, and there are also other generalizations of Postnikov towers. The generalization of Postnikov towers studied in this paper is the following, which generalizes the ones in [GM, Ma, Sm] and is included in the class of $[\mathbf{F}]$. Let us call the product of (possibly infinitely many) Eilenberg-Mac Lane spaces a generalized Eilenberg-Mac Lane space (GEM).

Received October 11, 2013, revised January 20, 2014; published on April 9, 2014.

2010 Mathematics Subject Classification: 55S45, 55P60.

Key words and phrases: Postnikov tower, generalized Eilenberg-Mac Lane space, localization, Postnikov length.

Article available at http://dx.doi.org/10.4310/HHA.2014.v16.n1.a8

Copyright (C) 2014, International Press. Permission to copy for private use granted. 
Definition 1.1. A generalized Postnikov tower (GPT) of a path-connected, finitetype space $X$ is a tower of fibrations

$$
\cdots \rightarrow X_{n} \rightarrow X_{n-1} \rightarrow \cdots \rightarrow X_{0}
$$

satisfying the following conditions:

1. $X_{0}$ is weakly contractible.

2. For $n \geqslant 1, X_{n}$ is the homotopy fiber of some map from $X_{n-1}$ to a simply connected, finite-type GEM.

3. There is a weak homotopy equivalence $X \rightarrow \lim _{\longleftarrow} X_{n}$.

We here remark two points in the above definition. The first point is the finite-type condition. This is standard in homotopy theory to control homotopy invariants. The second point is that the fibrations are special. In most interesting cases, each fibration in the classical Postnikov tower is principal; i.e., $X_{n}$ is the homotopy fiber of some map $X_{n-1} \rightarrow K\left(\pi_{n}(X), n+1\right)$. Then we assume that each fibration in a GPT is a principal fibration with the classifying map into a GEM.

A GPT is not a new object. There are several generalizations of Postnikov towers as in $[\mathbf{G M}, \mathbf{M a}, \mathbf{S m}, \mathbf{F}]$ that are closely related with a GPT, as is noted above. However, a GPT has not been studied systematically so far; it has been treated as a test example of well-developed tools like spectral sequences. The purpose of this paper is to study basic properties of a GPT such as its existence, localization, and length. We also consider a property of the length of a GPT of a finite complex as an analogy of the result of McGibbon of Neisendorfer [MN]. Finally, we give an algebraic description of the length of a GPT of a rational space.

Throughout this paper, we always assume the following. Every space is a pathconnected and finite-type (integrally or locally) CW-complex with a basepoint. Every map between spaces preserves basepoints.

\section{Existence}

In this section, we give a necessary and sufficient condition for the existence of a GPT of a given space. To this end, it is important to calculate the homotopy groups of a space from its GPT. Consider a GPT (1) of a space $X$. There is a tower of groups

$$
\cdots \rightarrow \pi_{k}\left(X_{n}\right) \rightarrow \pi_{k}\left(X_{n-1}\right) \rightarrow \cdots \rightarrow \pi_{k}\left(X_{0}\right) .
$$

As is well known, the homotopy groups of $X$ can be calculated from that of $X_{n}$ in the tower by the Milnor exact sequence

$$
1 \rightarrow \lim ^{1} \pi_{k+1}\left(X_{n}\right) \rightarrow \pi_{k}(X) \rightarrow \underset{\lim }{\longleftarrow} \pi_{k}\left(X_{n}\right) \rightarrow 1,
$$

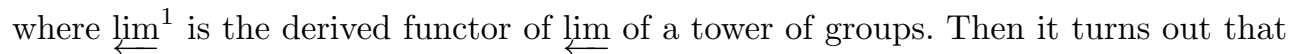
the control of $\lim ^{1} \pi_{k}\left(X_{n}\right)$ is important for our purpose. 
Let us recall here the definition of $\lim ^{1}$ of a tower of groups. Consider a tower of groups

$$
\cdots \stackrel{\alpha_{n+1}}{\longrightarrow} G_{n} \stackrel{\alpha_{n}}{\longrightarrow} G_{n-1} \stackrel{\alpha_{n-1}}{\longrightarrow} \cdots \stackrel{\alpha_{1}}{\longrightarrow} G_{0} .
$$

From this tower, we define an action of the group $\prod_{n \geqslant 0} G_{n}$ onto itself by

$$
\left(g_{0}, g_{1}, \ldots\right) \cdot\left(h_{0}, h_{1}, \ldots\right):=\left(h_{0} g_{0} \alpha_{1}\left(h_{1}\right)^{-1}, h_{1} g_{1} \alpha_{2}\left(h_{2}\right)^{-1}, \ldots\right)
$$

for $g_{n}, h_{n} \in G_{n}$. By definition, $\lim _{n}$ is the isotropy subgroup of the unit $(1,1, \ldots) \in$ $\prod_{n \geqslant 0} G_{n}$ with respect to this group action. Recall that $\lim ^{1} G_{n}$ is defined as the orbit space of this group action. Then, in particular, $\lim ^{1} G_{n}$ is just a pointed set in general, but it is an abelian group if all $G_{n}$ are abelian. The following lemma is useful in calculating $\lim ^{1}$.

Lemma 2.1 ([Mc, Theorem 4.2]). Suppose there is a commutative diagram of abelian groups

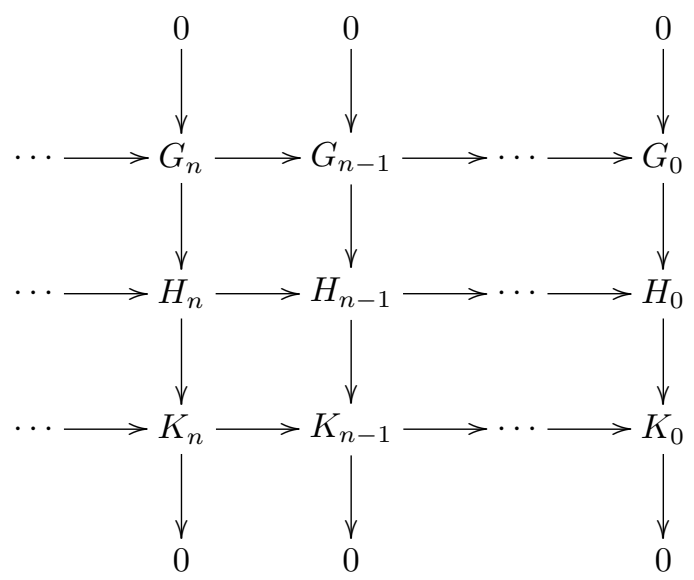

in which each column is exact. Then there is an exact sequence

$$
0 \rightarrow \underset{\lim }{\longleftarrow} G_{n} \rightarrow \lim _{\longleftarrow} H_{n} \rightarrow \underset{\lim }{\longleftarrow} K_{n} \rightarrow \lim ^{1} G_{n} \rightarrow \lim ^{1} H_{n} \rightarrow \lim ^{1} K_{n} \rightarrow 0 .
$$

Let us next recall the criteria for the triviality of $\varliminf^{1}$ of a tower of groups. There is a well-known criterion for the triviality of $\lim ^{1}$ called the Mittag-Leffler condition. In order to explain this condition, we set the notation. For a tower of groups (3), we put

$$
G_{n}^{(m)}:=\operatorname{Im}\left\{\alpha_{n+1} \circ \cdots \circ \alpha_{m}: G_{m} \rightarrow G_{n}\right\} \quad \text { for } m>n \quad \text { and } \quad G_{n}^{(\infty)}:=\bigcap_{m>n} G_{n}^{(m)} .
$$

A tower (3) is said to satisfy the Mittag-Leffler condition if the descending chain $G_{n}^{(n+1)} \supset G_{n}^{(n+2)} \supset \cdots$ stabilizes in finite steps for each $n$.

Lemma 2.2. If a tower (3) satisfies the Mittag-Leffler condition, $\lim ^{1} G_{n}$ is trivial.

In some cases, the converse to Lemma 2.2 holds as follows: 
Lemma 2.3 (McGibbon and Møller [MM]). Suppose that every $G_{n}$ is countable in a tower (3). Then $\lim ^{1} G_{n}$ is trivial if and only if (3) satisfies the Mittag-Leffler condition. Moreover, if $\lim ^{1} G_{n}$ is not trivial, then it is uncountable.

If all $\alpha_{n}$ in (3) are surjective, it is Mittag-Leffler, and hence $\lim ^{1} G_{n}$ is trivial. In this special case, we have the following, which can be proved analogously to showing that 2 -adic integers are uncountably many.

Lemma 2.4. If every $\alpha_{n}$ is surjective in a tower (3) and $\lim G_{n}$ is countable, then $\alpha_{N}$ is an isomorphism for $N$ large enough.

Recall from [HMR] that a space $X$ is called nilpotent if the action of $\pi_{1}(X)$ on $\pi_{k}(X)$ is nilpotent for any $k$. We now state the main theorem of this section.

Theorem 2.5. A space admits a GPT if and only if it is nilpotent.

Proof. If $X$ is nilpotent, its classical Postnikov tower has a principal refinement in the sense of $[\mathbf{H M R}]$, where the resulting tower is a GPT of $X$. Then the "if" part is proved.

Suppose that $X$ has a GPT (1). Let us first calculate $\pi_{1}(X)$. By definition, there is a homotopy fiber sequence

$$
X_{n} \rightarrow X_{n-1} \rightarrow K_{n}
$$

for each $n$, where $K_{n}$ is a simply connected GEM. Then the induced map $\pi_{1}\left(X_{n}\right) \rightarrow$ $\pi_{1}\left(X_{n-1}\right)$ is surjective, implying that there is a central extension

$$
0 \rightarrow A_{n} \rightarrow \pi_{1}\left(X_{n}\right) \rightarrow \pi_{1}\left(X_{n-1}\right) \rightarrow 1,
$$

where $A_{n}$ is a subgroup of $\pi_{2}\left(K_{n}\right)$. On the other hand, since we are now assuming that $X$ is of finite type, $\pi_{1}(X)$ is countable. Then it follows from Lemma 2.4 that the induced map $\pi_{1}\left(X_{N}\right) \rightarrow \pi_{1}\left(X_{N-1}\right)$ is an isomorphism for $N$ large enough, implying that the above central extension is trivial except for finitely many $n$. Hence we obtain that $\pi_{1}\left(X_{n}\right)$ is nilpotent.

Let us next consider the action of $\pi_{1}(X)$ on $\pi_{k}(X)$ for $k \geqslant 2$. Put $G_{n}=\pi_{k}\left(X_{n}\right)$. Since there is a homotopy fiber sequence (4) for each $n$, the action of $\pi_{1}\left(X_{n}\right)$ on $G_{n}$ is nilpotent. Then since $\pi_{1}\left(X_{N}\right) \cong \pi_{1}(X)$ for $N$ large enough, $G_{N}$ is a nilpotent $\pi_{1}(X)$-module for $N$ large enough. Hence we get a tower of nilpotent $\pi_{1}(X)$-modules

$$
\cdots \rightarrow G_{n} \rightarrow G_{n-1} \rightarrow \cdots \rightarrow G_{N} .
$$

Since we are assuming that each $X_{n}$ and $X$ are of finite type, $G_{n}$ and $\pi_{k}(X)$ are countable. Then by Lemma 2.3, the above tower satisfies the Mittag-Leffler condition, hence by Lemma 2.2 we obtain an isomorphism

$$
\pi_{k}(X) \stackrel{\cong}{\longrightarrow} \lim _{\longleftarrow} G_{n},
$$

which is compatible with the $\pi_{1}(X)$-action. Notice that $G_{N}^{(\infty)}$ is also a nilpotent $\pi_{1}(X)$-module for $N$ large enough. Then there is the tower of nilpotent $\pi_{1}(X)$-modules

$$
\cdots \rightarrow G_{n}^{(\infty)} \rightarrow G_{n-1}^{(\infty)} \rightarrow \cdots \rightarrow G_{N}^{(\infty)}
$$

in which every arrow is surjective since the tower (5) satisfies the Mittag-Lefler condition. Then since $\lim _{\longleftarrow} G_{n}^{(\infty)} \subset \lim _{\longleftarrow} G_{n} \cong \pi_{k}(X)$ is countable, we may assume that every 
arrow in this tower is an isomorphism, implying that $\lim _{\leftarrow} G_{n}^{(\infty)}=G_{N}^{(\infty)}$ is a nilpotent $\pi_{1}(X)$-module. Consider the third tower of abelian groups

$$
\cdots \rightarrow G_{n} / G_{n}^{(\infty)} \rightarrow G_{n-1} / G_{n-1}^{(\infty)} \rightarrow \cdots \rightarrow G_{N} / G_{N}^{(\infty)} .
$$

Since the tower (5) satisfies the Mittag-Leffler condition, for any $m \geqslant N$ there exists $l \geqslant 0$ such that the composite $G_{m+l} / G_{m+l}^{(\infty)} \rightarrow G_{m+l-1} / G_{m+l-1}^{(\infty)} \rightarrow \cdots \rightarrow G_{m} / G_{m}^{(\infty)}$ is trivial, implying that $\lim _{n} G_{n} / G_{n}^{(\infty)}=0$. Then it follows from Lemma 2.1 that the natural map of $\pi_{1}(X)$-modules $\lim G_{n}^{(\infty)} \rightarrow \lim G_{n}$ is an isomorphism. Thus we have proved that $\pi_{k}(X)$ is a nilpotent $\pi_{1}(X)$-module.

\section{Localization}

Let $p$ be a prime or zero, where if $p=0$, " $p$-local" means "rational." Let us define a $p$-local GPT of a $p$-local space $X$ by replacing a simply connected GEM with a simply connected $p$-local GEM in the definition of a GPT in Section 1. Then in particular, we see that Theorem 2.5 also holds $p$-locally, and so when we consider a GPT of a ( $p$-local) space $X$, we implicitly assume that $X$ is nilpotent. The $p$-localization of a tower of spaces $\cdots \rightarrow X_{n} \rightarrow X_{n-1} \rightarrow \cdots \rightarrow X_{0}$ means the term-wise $p$-localization $\cdots \rightarrow\left(X_{n}\right)_{(p)} \rightarrow\left(X_{n-1}\right)_{(p)} \rightarrow \cdots \rightarrow\left(X_{0}\right)_{(p)}$. Now it is natural to ask whether the $p$ localization of a GPT of a space $X$ is a $p$-local GPT of $X_{(p)}$ or not. The goal of this section is to prove the following answer to this question.

Theorem 3.1. The p-localization of a GPT of $X$ is a p-local GPT of $X_{(p)}$.

We prepare the following lemma.

Lemma 3.2. Consider a tower of finitely generated abelian groups

$$
\cdots \rightarrow A_{n} \rightarrow A_{n-1} \rightarrow \cdots \rightarrow A_{0} .
$$

If the tower satisfies the Mittag-Leffler condition and $\lim A_{n}$ is also finitely generated, the natural map

$$
\left(\lim _{\longleftarrow} A_{n}\right)_{(p)} \rightarrow \underset{\lim }{\longleftarrow}\left(A_{n}\right)_{(p)}
$$

is an isomorphism.

Proof. As in the proof of Theorem 2.5, since the tower (6) satisfies the Mittag-Leffler condition, the natural map $\lim A_{n}^{(\infty)} \rightarrow \lim A_{n}$ is an isomorphism. Since $\lim A_{n}^{(\infty)}$ $\left(\cong \lim A_{n}\right)$ is finitely generated and hence countable, it follows from Lemma 2.4 that the map $A_{n}^{(\infty)} \rightarrow A_{n-1}^{(\infty)}$ is an isomorphism for $n$ large enough. Then the natural map $\left(\varliminf_{n} A_{n}^{(\infty)}\right)_{(p)} \rightarrow \varliminf_{\lim }\left(A_{n}^{(\infty)}\right)_{(p)}$ is an isomorphism. Moreover, since the tower (6) satisfies the Mittag-Leffler condition, we have $\left(\left(A_{n}\right)_{(p)}\right)^{(\infty)} \leftarrow\left(A_{n}^{(\infty)}\right)_{(p)}$ is an isomorphism, and hence $\lim \left(\left(A_{n}\right)_{(p)}\right)^{(\infty)} \leftarrow \lim \left(A_{n}^{(\infty)}\right)_{(p)}$. On the other hand, since the tower (6) satisfies the Mittag-Leffler condition, so does its $p$-localization. Thus there is a 
commutative diagram of natural maps

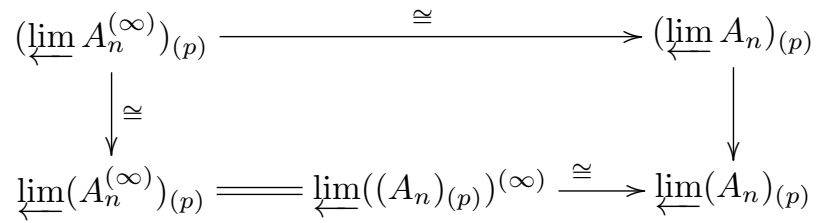

that implies the desired result.

Proof of Theorem 3.1. Let $\rho: X \rightarrow \lim _{\longleftarrow} X_{n}$ be a weak homotopy equivalence. By Lemma 3.2, the composite

$$
\pi_{k}(X)_{(p)} \stackrel{\left(\rho_{*}\right)_{(p)}}{\longrightarrow}\left(\lim _{\longleftarrow} \pi_{k}\left(X_{n}\right)\right)_{(p)} \rightarrow \underset{\lim }{\longleftarrow} \pi_{k}\left(X_{n}\right)_{(p)}
$$

is an isomorphism for $k \geqslant 2$, where the second arrow is the natural map. Then by the naturality of the $p$-localization, we get a map $\bar{\rho}: X_{(p)} \rightarrow \lim _{(}\left(X_{n}\right)_{(p)}$ that induces an isomorphism in $\pi_{k}$ for $k \geqslant 2$. As in the proof of Theorem 2.5, the induced tower $\cdots \rightarrow \pi_{1}\left(X_{n}\right) \rightarrow \pi_{1}\left(X_{n-1}\right) \rightarrow \cdots \rightarrow \pi_{1}\left(X_{0}\right)$ stabilizes in finite steps, implying that $\bar{\rho}$ induces an isomorphism also in $\pi_{1}$, completing the proof.

\section{Length}

Let $\cdots \rightarrow Y_{n} \rightarrow Y_{n-1} \rightarrow \cdots \rightarrow Y_{0}$ be a tower of fibrations. We define the length of this tower as the minimum $k$ such that for each $m>k$ the map $Y_{m} \rightarrow Y_{m-1}$ is a weak homotopy equivalence.

Definition 4.1. The Postnikov length of a space $X$, denoted by $\operatorname{PL}(X)$, is the minimum $n$ such that $X$ has a GPT of length $n$.

The purpose of this section is to collect basic properties of the Postnikov length. Of course, the Postnikov length is a homotopy invariant. By definition, $\operatorname{PL}(X)=0$ if and only if $X$ is weakly contractible, and $\operatorname{PL}(X)=1$ if and only if $X$ is a nontrivial GEM.

Let us first prove the following useful lemma. Let $X\langle n\rangle$ denote the $n$-connected cover of a space $X$, and let $\pi: X\langle n\rangle \rightarrow X$ be the natural projection. For a graded abelian group $A=\bigoplus_{n \geqslant 1} A_{n}$, let $K(A)$ denote the generalized Eilenberg-Mac Lane space $\prod_{n \geqslant 1} K\left(A_{n}, n\right)$.

Lemma 4.2. Suppose that a space $X$ has a GPT (1). Then there is a GPT

$$
\cdots \rightarrow X_{n}\langle m\rangle \rightarrow X_{n-1}\langle m\rangle \rightarrow \cdots \rightarrow X_{0}\langle m\rangle
$$

of $X\langle m\rangle$ in which each arrow is a part of a homotopy fibration $X_{n}\langle m\rangle \rightarrow X_{n-1}\langle m\rangle \rightarrow$ $K_{n}$ for an m-connected GEM $K_{n}$.

Proof. Let $A$ be a graded abelian group. For a map $\alpha: Y \rightarrow K(A)$ and a positive 
integer $m$, we define a subgroup $B \subset A$ by

$$
B_{n}= \begin{cases}0 & n \leqslant m \\ \operatorname{Im}\left\{\alpha_{*}: \pi_{m+1}(Y) \rightarrow A_{m+1}\right\} & n=m+1 \\ A_{n} & n>m+1 .\end{cases}
$$

Let $\bar{\alpha}$ be the composite $Y\langle m\rangle \stackrel{\pi}{\rightarrow} Y \stackrel{\alpha}{\rightarrow} K(A)$. Since there is a homotopy fibration $K(B) \rightarrow K(A) \rightarrow K(A / B)$ and $Y\langle m\rangle$ is $m$-connected, the obstruction to lift $\bar{\alpha}$ to a map $Y\langle m\rangle \rightarrow K(B)$ is the cohomology class in $H^{m+1}\left(Y\langle m\rangle ; A_{m+1} / B_{m+1}\right)$ that is represented by the composite

$$
Y\langle m\rangle \stackrel{\bar{\alpha}}{\rightarrow} K(A) \rightarrow K(A / B) \rightarrow K\left(A_{m+1} / B_{m+1}, m+1\right) .
$$

Then this obstruction can be identified with the composite $\pi_{m+1}(Y\langle m\rangle) \stackrel{\bar{\alpha}_{*}}{\longrightarrow} A_{m+1} \rightarrow$ $A_{m+1} / B_{m+1}$ through the congruences

$$
\begin{aligned}
H^{m+1}\left(Y\langle m\rangle ; A_{m+1} / B_{m+1}\right) & \cong \operatorname{Hom}\left(H_{m+1}(Y\langle m\rangle ; \mathbb{Z}), A_{m+1} / B_{m+1}\right) \\
& \cong \operatorname{Hom}\left(\pi_{m+1}(Y\langle m\rangle), A_{m+1} / B_{m+1}\right) .
\end{aligned}
$$

Then by the definition of $B$, the obstruction is trivial, and hence there is a map $\tilde{\alpha}: Y\langle m\rangle \rightarrow K(B)$ satisfying a homotopy commutative diagram

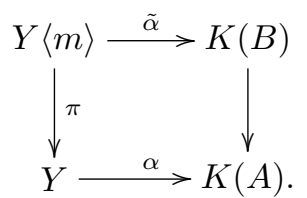

Let $F, \widetilde{F}$ be the homotopy fibers of the maps $\alpha, \tilde{\alpha}$, respectively. It is straightforward to verify that $\widetilde{F}$ is the $m$-connected cover of $F$ and that the natural map $\widetilde{F} \rightarrow F$ is idenfied with the projection $\pi: F\langle m\rangle \rightarrow F$.

Apply the above construction to the classifying map $X_{n-1} \rightarrow \bar{K}_{n}$ of the fibration $X_{n} \rightarrow X_{n-1}$, where $\bar{K}_{n}$ is a simply connected GEM. Then the proof of the first half of the lemma is proved, and it remains to show that there is a weak homotopy equivalence $X\langle m\rangle \rightarrow \lim X_{n}\langle m\rangle$. Obvoiusly, the composite $X\langle m\rangle \stackrel{\pi}{\rightarrow} X \stackrel{\rho}{\rightarrow} \lim X_{n}$ lifts to $\lim X_{n}\langle m\rangle$ through $\lim \pi: \lim X_{n}\langle m\rangle \rightarrow \lim X_{n}$, where $\rho$ is a weak homotopy equiv-

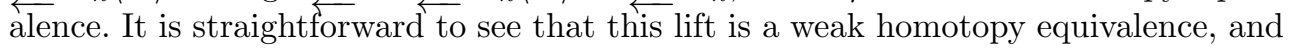
therefore the proof is completed.

The following proposition is an immediate consequence of Lemma 4.2.

Proposition 4.3. For any positive integer $m$, there is an inequality

$$
\operatorname{PL}(X\langle m\rangle) \leqslant \operatorname{PL}(X) .
$$

We consider the behavior of the Postnikov length on a fibration.

Proposition 4.4. For a fibration $F \rightarrow E \rightarrow B$ with a 2-connected base $B$, we have

$$
\mathrm{PL}(F) \leqslant \mathrm{PL}(E)+\mathrm{PL}(B) .
$$


Proof. Take a GPT $\cdots \rightarrow B_{n} \rightarrow B_{n-1} \rightarrow \cdots \rightarrow B_{0}$ of $B$ with length $k$. By Lemma 4.2, without raising length, we may assume that each $B_{n}$ is 2-connected and that each map is a part of a homotopy fibration $B_{n} \rightarrow B_{n-1} \rightarrow K_{n}$ with a 2-connected GEM $K_{n}$. We also take a GPT $\cdots \rightarrow E_{n} \rightarrow E_{n-1} \rightarrow \cdots \rightarrow E_{0}$ of $E$ with length $l$. Let $F_{i}$ be the homotopy fiber of the composite

$$
E_{l} \stackrel{\simeq}{\rightarrow} E \rightarrow B \stackrel{\simeq}{\rightarrow} B_{k} \rightarrow B_{k-1} \rightarrow \cdots \rightarrow B_{i}
$$

for $i \geqslant 1$. Notice that $F_{k} \simeq F$. It is well known that there is a homotopy commutative diagram

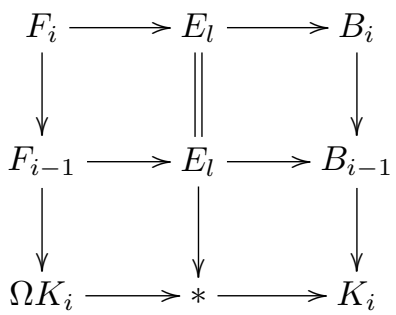

in which all rows and columns are homotopy fibrations. We are now assuming that each $K_{i}$ is a 2-connected GEM, and hence so is $B_{1}$. Then the tower

$$
\cdots=F=F_{k} \rightarrow F_{k-1} \rightarrow \cdots \rightarrow F_{1} \rightarrow E_{l} \rightarrow E_{l-1} \rightarrow \cdots \rightarrow E_{0}
$$

is a GPT of $F$ with length $l+k$, completing the proof.

Corollary 4.5. If $X$ is 2-connected,

$$
\operatorname{PL}(\Omega X) \leqslant \operatorname{PL}(X) .
$$

Proof. Apply Proposition 4.4 to the path-loop fibration of $X$.

It is useful to estimate the Postnikov length by other homotopy invariants. Recall that the Whitehead length of a space $X$, denoted by $\mathrm{WL}(X)$, is the minimum $n$ such that for some $x_{i} \in \pi_{k_{i}}(X)(i=1, \ldots, n+1)$ the iterated Whitehead product $\left[x_{1},\left[x_{2},\left[\cdots\left[x_{n}, x_{n+1}\right] \cdots\right]\right]\right.$ is nontrivial.

Proposition 4.6. For a space $X$, we have

$$
\mathrm{WL}(X) \leqslant \mathrm{PL}(X) .
$$

Proof. The proof of [BG, Theorem 3.3] implies that for a principal fibration $E \rightarrow B$, it holds that $\mathrm{WL}(E) \leqslant \mathrm{WL}(B)+1$, completing the proof.

We can consider the $p$-local version of the Postnikov length. The $p$-local Postnikov length of a $p$-local space $X$, denoted by $\mathrm{PL}_{(p)}(X)$, is defined as the minimum $n$ such that $X$ has a $p$-local GPT of length $n$. All of the above results in this section hold also for the $p$-local Postnikov length. The integral and the $p$-local Postnikov lengths are related by the following inequality, which is a consequence of Theorem 3.1. 
Corollary 4.7. For a space $X$, we have

$$
\mathrm{PL}_{(p)}\left(X_{(p)}\right) \leqslant \operatorname{PL}(X)
$$

To end this section, we give an interesting example of a space with finite Postnikov length, into which there is an essential phantom map but not after $p$-localization. Let us first recall the definition of phantom maps, where our reference for phantom maps is [Mc]. A map $f: X \rightarrow Y$ between spaces is called a phantom map if for any map $g: K \rightarrow X$ from a finite complex $K$, the composite $f \circ g$ is null homotopic. So $f$ is a phantom map if and only if the composite $X^{(n)} \rightarrow X \stackrel{f}{\rightarrow} Y$ is null homotopic for any $n$, where $X^{(n)}$ is the $n$-skeleton of $X$. Then by the homotopy set version of the Milnor exact sequence (2), there is an identification of all phantom maps from $X$ to $Y$, denoted by $\operatorname{Ph}(X, Y)$, as

$$
\operatorname{Ph}(X, Y) \cong \lim ^{1}\left[X^{(n)}, \Omega Y\right] .
$$

Let $\cdots \rightarrow Y_{n} \rightarrow Y_{n-1} \rightarrow \cdots \rightarrow Y$ be the classical Postnikov tower of $Y$. Then dually, we also have the identification

$$
\operatorname{Ph}(X, Y) \cong \lim ^{1}\left[X, \Omega Y_{n}\right] .
$$

In particular, if $Y$ is a finite Postnikov piece - i.e., the length of the classical Postnikov tower is finite - there is no essential phantom map into $Y$.

We now introduce the main object of this section. Let $\mathcal{P}$ be the set of all primes, and let $\mathcal{P}_{p}^{1}: K(\mathbb{Z}, 3) \rightarrow K(\mathbb{Z} / p, 2 p+1)$ be the composite of the modulo $p$ reduction $K(\mathbb{Z}, 3) \rightarrow K(\mathbb{Z} / p, 3)$ and the Steenrod reduced power operation $\mathcal{P}^{1}: K(\mathbb{Z} / p, 3) \rightarrow$ $K(\mathbb{Z} / p, 2 p+1)$, where $\mathcal{P}^{1}=\mathrm{Sq}^{2}$ for $p=2$. Define a space $X$ as the homotopy fiber of the map

$$
\rho=\prod_{p \in \mathcal{P}} \mathcal{P}_{p}^{1}: K(\mathbb{Z}, 3) \rightarrow \prod_{p \in \mathcal{P}} K(\mathbb{Z} / p, 2 p+1) .
$$

Obviously, we have $\operatorname{PL}(X) \leqslant 2$. By looking at cohomology, we also see that $\operatorname{PL}(X) \geqslant 2$; hence

$$
\operatorname{PL}(X)=2 \text {. }
$$

Notice that since $\rho$ is a stable cohomology operation, $X$ is an infinite loop space.

Theorem 4.8. For the above space $X$, we have

$$
\operatorname{Ph}(K(\mathbb{Z}, 2), X) \cong \mathbb{Z}^{\wedge} / \mathbb{Z} \oplus \bigoplus_{p \in \mathcal{P}} \mathbb{Z} / p^{\infty} \quad \text { and } \quad \operatorname{Ph}\left(K(\mathbb{Z}, 2), X_{(p)}\right)=*
$$

for any prime $p$, where $\mathbb{Z}^{\wedge}$ is the profinite completion of $\mathbb{Z}$. 
Proof. Consider the commutative diagram

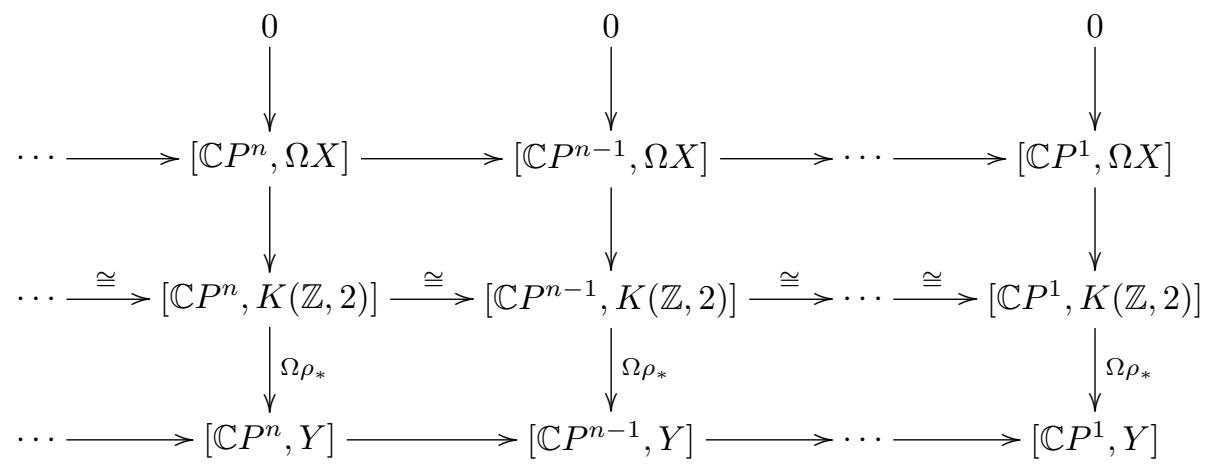

induced from the fibration $X \longrightarrow K(\mathbb{Z}, 2) \stackrel{\rho}{\longrightarrow} \prod_{p \in \mathcal{P}} K(\mathbb{Z} / p, 2 p+1)$, where $Y=$ $\prod_{p \in \mathcal{P}} K(\mathbb{Z} / p, 2 p)$. Let $u$ be the element of $[K(\mathbb{Z}, 2), K(\mathbb{Z} / p, 2)]$ corresponding to the modulo $p$ reduction. By the definition of the map $\rho$, we have

$$
(\Omega \rho)_{*}(u)=\left(u^{2}, u^{3}, \ldots, u^{p}, \ldots\right) \in[K(\mathbb{Z}, 2), Y],
$$

and hence the top row is identified with the tower

$$
\cdots \stackrel{p_{n+1}}{\longrightarrow} \mathbb{Z} \stackrel{p_{n}}{\longrightarrow} \mathbb{Z} \stackrel{p_{n-1}}{\longrightarrow} \cdots \stackrel{p_{1}}{\longrightarrow} \mathbb{Z},
$$

where $p_{n}$ is the product of all primes $\leqslant n$. Thus the first half follows from the identification (7) and the calculation in $[\mathbf{M c}]$. The second half follows from the fact that $X_{(p)}$ is a finite Postnikov piece.

\section{GPT of a finite complex}

In this section, we study the length of a GPT of a finite complex, motivated by the result of McGibbon and Neisendorfer [MN] on the distribution of torsion in the homotopy groups of a finite complex, which is a generalization of the classical result of Serre $[\mathbf{S e}]$. Let us first recall these results.

Theorem 5.1 (Serre [Se] for $p=2$ and Umeda [U] for $p$ odd). If $X$ is a simply connected finite complex, then $\pi_{n}(X)_{(p)} \neq 0$ for infinitely many $n$.

Theorem 5.2 (McGibbon and Neisendorfer $[\mathbf{M N}]$ ). If $X$ is a simply connected finite complex, $\pi_{n}(X)_{(p)}$ has torsion for infinitely many $n$.

Notice that Theorem 5.1 can be restated in terms of the classical Postnikov tower as follows: The length of the classical Postnikov tower of a simply connected finite complex is infinite at any prime $p$. There is a GPT version of this result due to Grodal whose proof is based on consideration of unstable modules. We here give a short and simple proof using the following Zabrodsky lemma.

Lemma 5.3 (Zabrodsky $[\mathbf{Z}]$ ). For a homotopy fibration $F \rightarrow E \rightarrow B$, if $\operatorname{map}_{*}(F, X)$ is weakly contractible, the induced map $\operatorname{map}_{*}(B, X) \rightarrow \operatorname{map}_{*}(E, X)$ is a weak homotopy equivalence. 
Theorem 5.4 (Grodal $[\mathbf{G r}]$ ). If $X$ is a simply connected $p$-locally finite complex that is not contractible, then $\mathrm{PL}_{(p)}(X)=\infty$.

Proof. Let $\operatorname{map}_{*}(K, X)$ denote the space of basepoint-preserving maps from $K$ to $X$. By the solution of the Sullivan conjecture on the homotopy fixed point set, the $p$ completion $\operatorname{map}_{*}(K, X)_{p}^{\wedge}$ is weakly contractible for any simply connected EilenbergMac Lane space $K$. Then it follows from Lemma 5.3 together with the natural homotopy equivalence $\operatorname{map}_{*}(K, X)_{p}^{\wedge} \simeq \operatorname{map}_{*}\left(K_{p}^{\wedge}, X_{p}^{\wedge}\right)$ that $\operatorname{map}_{*}(K, X)_{p}^{\wedge}$ is also weakly contractible for any simply connected space $K$ with finite Postnikov length. In particular, if we assume $\mathrm{PL}_{(p)}(X)<\infty, \operatorname{map}_{*}(X, X)_{p}^{\wedge}$ is weakly contractible. This implies that the identity map of $X_{p}^{\wedge}$ is null homotopic, and hence $X_{p}^{\wedge}$ is contractible, which is a contradiction.

As in the case of Theorem 5.1, we can restate Theorem 5.2 in terms of the classical Postnikov tower as follows: the homotopy fiber of the natural map $X \rightarrow X_{n}$ has torsion in the homotopy groups for any $n$, where $X_{n}$ is the $n$-th Postnikov piece. So we consider the following problem as a generalization of Theorem 5.2 using a GPT.

Problem 5.5. Consider a GPT (1) of a simply connected finite complex $X$. Do the homotopy groups of the homotopy fiber of the natural map $X \rightarrow X_{n}$ have $p$-torsion for any $n$ ?

We give an affirmative answer to this problem in several cases. Let us first consider the case $\mathrm{WL}(X)=\infty$. We prepare the following variant of the argument in $[\mathbf{M N}]$.

Lemma 5.6. Suppose that a map $f: X \rightarrow Y$ between p-local nilpotent spaces satisfies the following conditions:

1. $f_{*}: \pi_{m}(X) \rightarrow \pi_{m}(Y)$ is nontrivial for some $m \geqslant 3$.

2. $\pi_{n}(X)$ is torsion-free for any $n$.

Then $Y$ cannot be a simply connected p-locally finite complex.

Proof. By assumption, there is a map $g_{1}: \mathbb{C} P^{1} \rightarrow \Omega^{m-2} X$ for some $m \geqslant 3$ such that $\Omega^{m-2} f \circ g_{1}$ is essential. Suppose that $g_{1}$ extends to a map $g_{n}: \mathbb{C} P^{n} \rightarrow \Omega^{m-2} X$. We consider the induced map of $g_{n}$ in the minimal models of rational homotopy theory. Since every rational H-space is a GEM, the minimal model of $\Omega^{m-2} X$ is of the form $(\Lambda V, 0)$ for some graded vector space $V$. On the other hand, the minimal model of $\mathbb{C} P^{n}$ is given by

$$
(\Lambda(u, v), d), \quad|u|=2,|v|=2 n+1 \quad \text { and } \quad d v=u^{n+1} .
$$

Then we see that $g_{n}$ induces the trivial map in the minimal models. Then, in particular, the associated linear map $V=\Lambda V / \Lambda^{+} V \rightarrow \Lambda(u, v) / \Lambda^{+}(u, v)=\langle u, v\rangle$ is also trivial; this is identified with the induced map $\operatorname{Hom}\left(\left(g_{n}\right)_{*}, \mathbb{Q}\right): \operatorname{Hom}\left(\pi_{*}\left(\Omega^{m-2} X\right), \mathbb{Q}\right) \rightarrow$ $\operatorname{Hom}\left(\pi_{*}\left(\mathbb{C} P^{n}\right), \mathbb{Q}\right)$; see $\left[\right.$ FHT2]. Thus, as the rationalization map $\pi_{2 n+1}\left(\Omega^{m-2} X\right) \rightarrow$ $\pi_{2 n+1}\left(\Omega^{m-2} X\right)_{(0)}$ is injective by assumption, the map $\left(g_{n}\right)_{*}: \pi_{2 n+1}\left(\mathbb{C} P^{n}\right) \rightarrow$ $\pi_{2 n+1}\left(\Omega^{m-2} X\right)$ is trivial, implying that $g_{n}$ extends to $g_{n+1}: \mathbb{C} P^{n+1} \rightarrow \Omega^{m-2} X$.

Taking the (homotopy) colimit, we get a map $g_{\infty}: \mathbb{C} P^{\infty} \rightarrow \Omega^{m-2} X$ such that $\Omega^{m-2} f \circ g_{\infty}$ is essential, implying that $\operatorname{map}_{*}\left(\mathbb{C} P^{\infty}, Y\right)$ is not weakly contractible (after $p$-completion). By the solution to the Sullivan conjecture on the homotopy 
fixed point sets, this implies that $Y$ cannot be a simply connected $p$-locally finite complex. Therefore the proof is completed.

Theorem 5.7. Let $X$ be a simply connected p-locally finite complex with $\mathrm{WL}(X)=\infty$. Then for any $p$-local GPT (1) of $X$ and any $n$, the homotopy fiber of the map $X \rightarrow X_{n}$ has torsion in the homotopy groups.

Proof. Let $F$ be the homotopy fiber of the map $X \rightarrow X_{n}$. By the $p$-local version of Proposition 4.6, we have $\operatorname{WL}\left(X_{n}\right) \leqslant \operatorname{PL}_{(p)}\left(X_{n}\right) \leqslant n$. Then since $\operatorname{WL}(X)=\infty$ and $X$ is simply connected, the induced map $\pi_{m}(X) \rightarrow \pi_{m}\left(X_{n}\right)$ is not injective for some $m \geqslant 3$, implying that the induced map $\pi_{m}(F) \rightarrow \pi_{m}(X)$ is nontrivial for some $m \geqslant 3$. Assume that $\pi_{m}(F)$ is torsion-free for any $m$. Then by Lemma $5.6, X$ cannot be a simply connected $p$-locally finite complex, a contradiction. Thus $\pi_{*}(F)$ has torsion.

Recall that a simply connected space $X$ is called rationally elliptic if $\pi_{n}(X) \otimes \mathbb{Q} \neq 0$ for finitely many $n$. If $X$ is not rationally elliptic, it is called rationally hyperbolic; i.e., $\pi_{n}(X) \otimes \mathbb{Q} \neq 0$ for infinitely many $n$.

Corollary 5.8. Let $X$ be a simply connected p-locally finite complex with a p-local GPT (1). If $X$ is rationally hyperbolic, the homotopy fiber of the map $X \rightarrow X_{n}$ has torsion in the homotopy groups for any $n$.

Proof. It is proved in [FHT1] that if $X$ is rationally hyperbolic, $\operatorname{WL}(X)=\infty$. Thus the proof is completed by Theorem 5.7.

We next consider the case that the $v_{1}$-periodic homotopy groups are nontrivial. Let us recall the definition of the $v_{1}$-periodic groups, where we refer to $[\mathbf{D}]$ for more details. Let $M^{n}(p)$ denote the $n$-dimensional Moore space $S^{n-1} \cup_{p} e^{n}$. Recall that there is the so-called Adams map $\alpha: M^{n+s}(p) \rightarrow M^{n}(p)$ for $s=8,2 p-2$, according as $p=2$ and $p>2$. The $v_{1}$-periodic homotopy group of a space $X$, denoted by $v_{1}^{-1} \pi_{n}(X ; \mathbb{Z} / p)$, is defined as the colimit of the sequence

$$
\left[M^{n}(p), X\right] \stackrel{\alpha^{*}}{\longrightarrow}\left[M^{n+s}(p), X\right] \stackrel{\alpha^{*}}{\longrightarrow}\left[M^{n+2 s}(p), X\right] \stackrel{\alpha^{*}}{\longrightarrow} \cdots .
$$

For a fibration $F \rightarrow E \rightarrow B$, we have a homotopy exact sequence [D]

$\cdots \rightarrow v_{1}^{-1} \pi_{n}(F ; \mathbb{Z} / p) \rightarrow v_{1}^{-1} \pi_{n}(E ; \mathbb{Z} / p) \rightarrow v_{1}^{-1} \pi_{n}(B ; \mathbb{Z} / p) \rightarrow v_{1}^{-1} \pi_{n-1}(F ; \mathbb{Z} / p) \rightarrow \cdots$

There are several examples of finite complexes with nontrivial $v_{1}$-periodic homotopy groups such as spheres and compact Lie groups $[\mathbf{D}]$.

Theorem 5.9. Let $X$ be a simply connected p-locally finite complex with a p-local GPT (1). If $v_{1}^{-1} \pi_{*}(X ; \mathbb{Z} / p) \neq 0$, the homotopy fiber of the map $X \rightarrow X_{n}$ has torsion in the homotopy groups for any $n$.

Proof. For any GEM $K$, every arrow in the sequence (8) becomes trivial after finitely many steps, so we have $v_{1}^{-1} \pi_{n}(K ; \mathbb{Z} / p)=0$. Then by the homotopy exact sequence, we get that $v_{1}^{-1} \pi_{n}(X ; \mathbb{Z} / p)=0$ for a $p$-local space with $\mathrm{PL}_{(p)}(X)<\infty$.

Let $F$ be the homotopy fiber of the map $X \rightarrow X_{n}$ with the fiber inclusion $i: F \rightarrow$ $X$. Since $v_{1}^{-1} \pi_{*}\left(X_{n} ; \mathbb{Z} / p\right)=0$, it follows from the homotopy exact sequence that the induced map $i_{*}: v_{1}^{-1} \pi_{n}(F ; \mathbb{Z} / p) \rightarrow v_{1}^{-1} \pi_{n}(X ; \mathbb{Z} / p)$ is an isomorphism for any $n$. Then, as $v_{1}^{-1} \pi_{*}(X ; \mathbb{Z} / p) \neq 0$ by assumption, for some integer $N$ the map $i^{*}:\left[M^{N}(p), F\right] \rightarrow$ 
$\left[M^{N}(p), X\right]$ is nontrivial. Consider the following commutative diagram induced from the cofibration sequence $S^{N-1} \stackrel{p}{\rightarrow} S^{N-1} \rightarrow M^{N}(p) \stackrel{\pi}{\rightarrow} S^{N}$.

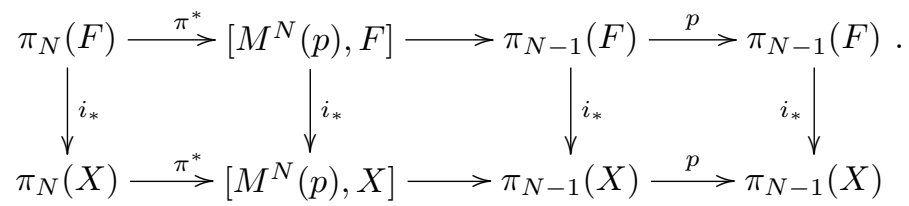

Suppose now that $\pi_{*}(F)$ has no torsion. Then the $p$-power map in the upper right of the diagram is injective, implying that the map $\pi^{*}: \pi_{N}(F) \rightarrow\left[M^{N}(p), F\right]$ in the upper left is surjective. Hence since the second vertical arrow $i_{*}:\left[M^{N}(p), F\right] \rightarrow\left[M^{N}(p), X\right]$ is nontrivial as above, we obtain that the first vertical arrow $i_{*}: \pi_{N}(F) \rightarrow \pi_{N}(X)$ is also nontrivial. Thus by Lemma 5.6, $X$ cannot be a simply connected $p$-locally finite complex, a contradiction. Therefore $\pi_{*}(F)$ has torsion, completing the proof.

Remark 5.10. Let $X$ be a 3 -connected space. It is proved in $[\mathbf{B}]$ that $v_{1}^{-1} \pi_{*}(X ; \mathbb{Z} / p) \neq$ 0 if and only if the mod $p K$-homology of $\left(\Omega^{n} X\right)\langle 3\rangle$ is nontrivial for any $n$. If $X$ is rationally hyperbolic, we have $H_{*}\left(\left(\Omega^{n} X\right)\langle 3\rangle ; \mathbb{Q}\right) \neq 0$ for any $n$, implying that the mod $p K$-homology of $\left(\Omega^{n} X\right)\langle 3\rangle$ is nontrivial for any $n$. Then Corollary 5.8 for 3 -connected spaces follows from Theorem 5.9.

We have considered the rationally hyperbolic case, so we next consider the rationally elliptic case. Let us recall the following result.

Theorem 5.11 (McGibbon and Wilkerson [MW]). Let $X$ be a simply connected finite complex that is rationally elliptic and is not rationally contractible. Then except for finitely many $p$, there is a p-local homotopy equivalence

$$
\Omega X \simeq_{(p)} \prod_{i=1}^{k} S^{2 m_{i}+1} \times \prod_{j=1}^{l} \Omega S^{2 n_{j}+1} .
$$

Corollary 5.12. Let $X$ be a simply connected finite complex that is rationally elliptic and is not rationally contractible, and let the tower (1) be a GPT of X. Except for finitely many $p$, the homotopy fiber of the map $X \rightarrow X_{n}$ has $p$-torsion in the homotopy groups for any $n$.

Proof. As is noted above, the $v_{1}$-periodic homotopy groups of spheres are nontrivial. Then by Theorem $5.11, v_{1}^{-1} \pi_{*}(X ; \mathbb{Z} / p) \neq 0$ except for finitely many $p$, and hence the proof is completed by Theorem 5.9.

\section{Rational Postnikov length}

As is well known, the rational homotopy type of a simply connected space is completely described by the dga called the minimal model. According to this, many homotopy invariants are now described in terms of the minimal model, or a Sullivan model in general. The purpose of this section is to give an algebraic description of the rational Postnikov length. Our reference for rational homotopy theory is [FHT2].

We first recall basic terminology in rational homotopy theory. Throughout this section, vector spaces and dga's are over the field of rational numbers $\mathbb{Q}$. Let $V$ be a 
graded vector space. A filtration of $V$ is an ascending chain $0=V(0) \subset V(1) \subset \cdots$ of vector subspaces of $V$ satisfying $\bigcup_{n \geqslant 0} V(n)=V$. The length of a Sullivan filtration is the minimum $k$ such that $V(k)=V$. Recall that a dga $(\Lambda V, d)$ is called a Sullivan algebra if there is a filtration $0=V(0) \subset V(1) \subset \cdots$ of $V$ satisfying

$$
d V(n) \subset \Lambda V(n-1) \quad \text { for all } n .
$$

Such a filtration is called a Sullivan filtration. A Sullivan algebra $(\Lambda V, d)$ is called minimal if $d v$ is decomposable for any $v \in V$. A map between dga's is called a quasiisomorphism if it induces an isomorphism in cohomology. We have the following fundamental theorem in the algebraic part of rational homotopy theory.

Theorem 6.1 (cf. [FHT2]). Let $(A, d)$ be a simply connected dga.

1. There is a Sullivan algebra $(\Lambda V, d)$ and a quasi-isomorphism $\rho:(\Lambda V, d) \rightarrow(A, d)$. The dga $(\Lambda V, d)$ (or the map $\rho$ ) is called a Sullivan model of $(A, d)$.

2. The above Sullivan algebra can be chosen as minimal.

3. The minimal Sullivan model of $(A, d)$ is unique up to homotopy.

Let us define the algebraic Postnikov length of a dga.

Definition 6.2. The Postnikov length of a dga $(A, d)$, denoted by $\operatorname{PL}(A, d)$, is the minimum $n$ such that there is a Sullivan model of $(A, d)$ with filtration length $n$.

By definition, $\operatorname{PL}(A, d)=0$ if and only if $(A, d)$ is acyclic, and $\operatorname{PL}(A, d)=1$ if and only if $(A, d)$ is not acyclic and there is a Sullivan model of $(A, d)$ with a trivial differential. The Postnikov length of a dga is a quasi-isomorphism invariant as:

Proposition 6.3. For dga's $(A, d)$ and $(B, d)$, if there exists a quasi-isomorphism $(A, d) \rightarrow(B, d)$, then

$$
\mathrm{PL}(A, d)=\mathrm{PL}(B, d) .
$$

Proof. Obviously, we have $\operatorname{PL}(A, d) \geqslant \operatorname{PL}(B, d)$. Let $\rho:(\Lambda V, d) \rightarrow(B, d)$ be a Sullivan model of $(B, d)$. Then the map $\rho$ lifts to a map $\tilde{\rho}:(\Lambda V, d) \rightarrow(A, d)$ through the given quasi-isomorphism $(A, d) \rightarrow(B, d)$. Then $\tilde{\rho}$ is a Sullivan model of $(A, d)$, implying $\mathrm{PL}(A, d) \leqslant \operatorname{PL}(B, d)$.

For a dga $(\Lambda V, d)$, we define the special filtration $V[0] \subset V[1] \subset \cdots$ of $V$ as

$$
V[0]=0 \quad \text { and } \quad V[n]=\{v \in V \mid d v \in \Lambda V[n-1]\} \quad \text { for } n \geqslant 1 .
$$

Proposition 6.4. The length of the filtration $V[0] \subset V[1] \subset \cdots$ of $V$ is minimal among all Sullivan filtration of $(\Lambda V, d)$.

Proof. Let $V(0) \subset V(1) \subset \cdots$ be an arbitrary Sullivan filtration of $V$. By definition, we have $V(0)=V[0]=0$. Suppose that $V(n-1) \subset V[n-1]$. Then $d V(n) \in \Lambda V(n-$ 1) $\subset \Lambda V[n-1]$, implying that $V(n) \subset V[n]$. This completes the proof.

As in Theorem 6.1, the quasi-isomorphism type of a dga is determined by its minimal model. So it is natural to consider whether the Postnikov length of a dga can be described by its minimal model. This leads us to define the minimal model Postnikov length of a dga $(A, d)$, denoted by $\operatorname{PL}_{\min }(A, d)$, as the minimum $n$ such 
that there is the minimal model of $(A, d)$ that admits a length $n$ Sullivan filtration. By definition, we have

$$
\mathrm{PL}_{\min }(A, d) \geqslant \operatorname{PL}(A, d) \text {. }
$$

But unfortunately, the following example shows that the converse implication does not hold. Thus the rational Postnikov length cannot be determined only from the minimal Sullivan model.

Example 6.5. Consider a dga $(\Lambda V, d)$ with $V=\left\langle x_{3}, x_{5}, x_{7}, x_{17}, x_{31}, x_{47}, u_{9}, u_{10}\right\rangle$ and

$$
\begin{aligned}
& d x_{3}=d x_{5}=d x_{17}=0, \quad d x_{7}=x_{3} x_{5}, \quad d x_{31}=x_{5} x_{17} u_{10}, \quad d x_{47}=x_{17} x_{31}, \\
& d u_{9}=u_{10}+x_{3} x_{7}, \quad d u_{10}=0,
\end{aligned}
$$

where the suffix indicates the degree. The minimum length Sullivan filtration of $V$ is given as

$$
V[0]=0, V[1]=\left\langle x_{3}, x_{5}, x_{17}, u_{10}\right\rangle, V[2]=V[1] \oplus\left\langle x_{7}, x_{31}, u_{9}\right\rangle, V[3]=V ;
$$

hence $\operatorname{PL}(\Lambda V, d)=3$. The minimal model of $(\Lambda V, d)$ is a dga $(\Lambda W, d)$ with $W=$ $\left\langle y_{3}, y_{5}, y_{7}, y_{17}, y_{31}, y_{47}\right\rangle$ and

$$
d y_{3}=d y_{5}=d y_{17}=0, d y_{7}=y_{3} y_{5}, d y_{31}=y_{3} y_{5} y_{7} y_{17}, d y_{47}=y_{17} y_{31},
$$

where the quasi-isomorphism $\rho:(\Lambda W, d) \rightarrow(\Lambda V, d)$ is given by

$$
\rho\left(y_{i}\right)=x_{i} \text { for } i=3,5,7,17,47 \text { and } \rho\left(y_{31}\right)=x_{31}-x_{5} x_{17} u_{9} .
$$

The minimum length Sullivan filtration of $(\Lambda W, d)$ is

$$
\begin{aligned}
& W[0]=0, \quad W[1]=\left\langle y_{3}, y_{5}, y_{17}\right\rangle, \quad W[2]=W[1] \oplus\left\langle y_{7}\right\rangle, \\
& W[3]=W[2] \oplus\left\langle y_{31}\right\rangle, \quad W[4]=W .
\end{aligned}
$$

For a degree reason, the minimal model $(\Lambda W, d)$ is unique, and then $\mathrm{PL}_{\min }(\Lambda V, d)=4$.

For a space $X$, there is a dga $A_{\mathrm{PL}}(X)$ consisting of PL de Rham forms whose cohomology is naturally isomorphic to $H^{*}(X ; \mathbb{Q})$. Then by Theorem 6.1 , the rational homotopy type of a simply connected space $X$ is completely determined by the minimal model of $A_{\mathrm{PL}}(X)$. The functor $A_{\mathrm{PL}}$ has a left adjoint that is denoted by $|(A, d)|$ for a dga $(A, d)$.

We consider the algebraic object corresponding to a fibration. Recall that a KSextension of a dga $(A, d)$ by a graded vector space $V$ is a dga $(A \otimes \Lambda V, \bar{d})$ satisfying

$$
\left.\bar{d}\right|_{A}=d \quad \text { and } \quad d V \subset A .
$$

Let $\Sigma V$ be the graded vector space with $\Sigma V_{n}=V_{n-1}$. Then the differential of the above KS-extension corresponds to a map $(\Sigma V, 0) \rightarrow(A, d)$. We know that for a KSextension $(A \otimes \Lambda V, \bar{d})$ of a dga $(A, d)$ defined by a map $(\Sigma V, 0) \rightarrow(A, d)$, the natural sequence $(A, d) \rightarrow(A \otimes \Lambda V, \bar{d}) \rightarrow(\Lambda V, 0)$ induces a principal fibration

$$
|(\Lambda V, 0)| \rightarrow|(A \otimes \Lambda V, \bar{d})| \rightarrow|(A, d)|
$$

that is classified by the induced map $|(A, d)| \rightarrow|(\Lambda \Sigma V, 0)|$. Moreover, we also know that for a graded vector space $W,|(\Lambda W, 0)|$ is the GEM $K(W)$.

The converse of the above construction also holds as follows. Let $E \rightarrow B$ be a principal fibration that is classified by a map $B \rightarrow K(\Sigma W)$ for a graded vector space $W$. 
Let $(A, d) \rightarrow A_{\mathrm{PL}}(B)$ be a quasi-isomorphism. The minimal model of $A_{\mathrm{PL}}(K(\Sigma W))$ is $(\Lambda \Sigma W, 0)$, and the composite $(\Lambda \Sigma W, 0) \rightarrow A_{\mathrm{PL}}(K(\Sigma W)) \rightarrow A_{\mathrm{PL}}(B)$ lifts to $(A, d)$ through the given quasi-isomorphism. Then we get a KS-extension $(A \otimes \Lambda W, \bar{d})$. As in [FHT2], there is a commutative diagram

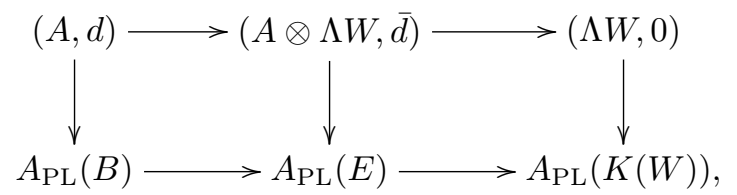

where the bottom row is induced from a fibration $K(W) \rightarrow E \rightarrow B$ and each vertical arrow is a quasi-isomorphism.

We are now ready to show a relation between the Postnikov length of a space $X$ and the Postnikov length of the dga $A_{\mathrm{PL}}(X)$.

Theorem 6.6. For a rational space $X$, we have

$$
\mathrm{PL}_{(0)}(X)=\operatorname{PL}\left(A_{\mathrm{PL}}(X), d\right) .
$$

Proof. Let $(\Lambda V, d)$ be a Sullivan model of $A_{\mathrm{PL}}(B)$. By (9), for the minimum Sullivan filtration $V[0] \subset V[1] \subset \cdots$ of $V$, there is a tower

$$
\cdots \rightarrow|(\Lambda V[n], d)| \rightarrow|(\Lambda V[n-1], d)| \rightarrow \cdots \rightarrow|(\Lambda V[0], d)|=*
$$

in which each arrow $|(\Lambda V[n], d)| \rightarrow|(\Lambda V[n-1], d)|$ is a principal fibration classified by a map $|(\Lambda V[n-1], d)| \rightarrow|(\Sigma(V[n] / V[n-1]), 0)|=K(\Sigma(V[n] / V[n-1]))$. Since the functor $A_{\mathrm{PL}}$ is a is a right adjoint, we have

$$
\lim _{\longleftarrow}|(\Lambda V[n], d)|=\left|\lim _{\longrightarrow}(\Lambda V[n], d)\right|=|(\Lambda V, d)| .
$$

Then the above tower is a GPT of $X$, implying

$$
\mathrm{PL}_{(0)}(X) \leqslant \operatorname{PL}\left(A_{\mathrm{PL}}(X), d\right) .
$$

Suppose $X$ has a rational GPT (1). Then each arrow $X_{n} \rightarrow X_{n-1}$ corresponds to a KS-extension for a given quasi-isomorphism $(A, d) \rightarrow A_{\mathrm{PL}}\left(X_{n-1}\right)$, and hence we get a sequence of KS-extensions

$$
0=(\Lambda V(0), d) \rightarrow \cdots \rightarrow(\Lambda V(n-1), d) \rightarrow(\Lambda V(n), d) \rightarrow \cdots
$$

such that there is a natural quasi-isomorphism $(\Lambda V(n), d) \rightarrow A_{\mathrm{PL}}\left(X_{n}\right)$. Since $\lim _{\mathrm{PL}}\left(X_{n}\right)=A_{\mathrm{PL}}\left(\lim _{\longleftarrow} X_{n}\right)$ as above, $(\Lambda V, d)=\underline{\lim }(\Lambda V(n), d)$ is a Sullivan model $\overrightarrow{\text { of }} A_{\mathrm{PL}}(X)$, implying

$$
\mathrm{PL}_{(0)}(X) \geqslant \operatorname{PL}\left(A_{\mathrm{PL}}(X), d\right) .
$$

Therefore, the proof is completed.

\section{Problems}

In this last section, we propose some problems concerning a GPT that naturally arise in the above study. We first consider a relation between the Postnikov length 
and localization. By Corollary 4.7, we have

$$
\operatorname{PL}(X) \geqslant \max \left\{\operatorname{PL}_{(p)}\left(X_{(p)}\right) \mid p \in \mathcal{P}\right\},
$$

where $\mathcal{P}$ is the set of all primes as above. However, we do not know whether the equality holds or not. Then we raise the following problem.

Problem 7.1. Does the equality in (11) always hold?

We next consider the distribution of torsion in a GPT of a finite complex. Let $X$ be a simply connected finite complex with a GPT (1). We here write the problem in Section 5 once again.

Problem 7.2. Consider a GPT (1) of a simply connected finite complex $X$. Do the homotopy groups of the homotopy fiber of the natural map $X \rightarrow X_{n}$ have $p$-torsion for any $n$ ?

We have answered to this problem affirmatively in many cases. In the proof of this answer, the key is to show the noninjectivity of the map $\pi_{*}(X) \rightarrow \pi_{*}\left(X_{n}\right)$. So we raise the following problem.

Problem 7.3. Let $X$ be a simply connected finite complex with a GPT (1). Is the induced map $\pi_{*}(X)_{(p)} \rightarrow \pi_{*}\left(X_{n}\right)_{(p)}$ noninjective for any $n$ ?

A slightly generalized problem is the following, which might be thought of as a "homotopy" version of the immersion problem.

Problem 7.4. Let $X$ be a simply connected finite complex, and let $Y$ be a $p$-local space with $\operatorname{PL}_{(p)}(Y)<\infty$. Does any map $X_{(p)} \rightarrow Y$ induce a noninjection in the homotopy groups?

Of course, there are implications:

$$
\text { Problem } 7.4 \Rightarrow \text { Problem } 7.3 \Rightarrow \text { Problem } 7.2
$$

We finally consider the rational Postnikov length. For simplicity, we only consider rational spaces. In $[\mathbf{Y}]$, Yau defines the fiber length of a space $X$, denoted by $\mathrm{FL}_{Y}(X)$, as follows. $\mathrm{FL}_{\mathrm{Y}}(X)=0$ if and only if $X$ is contractible, and $\mathrm{FL}_{Y}(X) \leqslant n$ if and only if there is a homotopy fibration $X \rightarrow Y \rightarrow Z$ in which $\mathrm{FL}_{Y}(Y)<n$. In $[\mathbf{H}]$, Hovey gives another definition of the fiber length of a space by adding the extra condition that $Z$ is an H-space in the above definition due to Yau. We denote Hovey's fiber length by $\mathrm{FL}_{\mathrm{H}}(X)$. Since every rational $\mathrm{H}$-space is a GEM, we have

$$
\mathrm{FL}_{\mathrm{H}}(X)=\mathrm{PL}_{(0)}(X) \text {. }
$$

By definition, we also have

$$
\mathrm{FL}_{Y}(X) \leqslant \mathrm{FL}_{\mathrm{H}}(X) \quad\left(=\mathrm{PL}_{(0)}(X)\right) .
$$

The fiber length is defined in connection with the cocategory, and there are two definitions of the cocategory $[\mathbf{G a}, \mathbf{H}]$ corresponding to the above two definitions of the fiber length. It is known that there is an inequality between these two cocateogories, and Hovey $[\mathbf{H}]$ proved that the inequality for rational spaces is strict in general. We now raise the following problem.

Problem 7.5. Is there a rational space $X$ for which the inequality (12) is strict? 


\section{References}

[B] A.K. Bousfield, Localization and periodicity in unstable homotopy theory, J. Amer. Math. Soc 7 (1994), no. 4, 831-873.

[BG] I. Berstein and T. Ganea, Homotopical nilpotency, Illinois J. Math. 5 (1961), no. 5, 1-174.

[D] D.M. Davis, Computing v v-periodic homotopy groups of spheres and certain Lie groups, Handbook of Algebraic Topology, Elsevier (1995), 993-1049.

[F] E.D. Farjoun, Cellular spaces, null spaces and homotopy localization, Lecture Notes in Mathematics 1622, Springer-Verlag, Berlin, 1996.

[FHT1] Y. Félix, S. Halperin, and J.-C. Thomas, The homotopy Lie algebra for finite complexes, Inst. Hautes Études Sci. Publ. Math 56 (1982), 179-202.

[FHT2] Y. Félix, S. Halperin, and J.-C. Thomas, Rational homotopy theory, Graduate Texts in Mathematics 205, Springer-Verlag, New York, 2001.

[Ga] T. Ganea, Lusternik-Schnirelmann category and cocategory, Proc. London Math. Soc. 10 (1960), no. 3, 623-639.

[GM] S. Gitler and M. Mahowald, The geometric dimension of real stable vector bundles, Bol. Soc. Mat. Mexicana 11 (1966), 85-107.

[Gr] J. Grodal, The transcendence degree of the mod $p$ cohomology of finite Postnikov systems, Stable and unstable homotopy (Toronto, ON, 1996), Fields Inst. Comm. 19, Amer. Math. Soc., Providence, RI, 1998, 111-130.

[HMR] P. Hilton, G. Mislin, and J. Roitberg, Localization of nilpotent groups and spaces, in North-Holland Mathematics Studies, vol. 15, Notas de Matemática (Notes on Mathematics), vol. 55, North-Holland Publishing Co./American Elsevier Publishing Co., Inc., Amsterdam, Oxford/New York, 1975.

[H] M. Hovey, Lusternik-Schnirelmann cocategory, Illinois J. Math. 37 (1993), 224-239.

[Ma] M. Mahowald, On obstruction theory in orientable fiber bundles, Trans. Amer. Math. Soc. 110 (1964), 315-349.

[Mc] C.A. McGibbon, Phantom maps, Chapter 25 in Handbook of Algebraic Topology, Elsevier (1995).

[MM] C.A. McGibbon and J.M. Møller, On spaces with the same n-type for all $n$, Topology 31 (1992), no. 1, 177-201.

[MN] C.A. McGibbon and J.A. Neisendorfer, On the homotopy groups of a finitedimensional space, Comment. Math. Helv. 59 (1984), no. 2, 253-257.

[MW] C.A. McGibbon and C.W. Wilkerson, Loop spaces of finite complexes at large primes, Proc. Amer. Math. Soc. 96 (1986), 698-702.

[Se] J.-P. Serre, Cohomologie modulo 2 des complexes d'Eilenberg-Mac Lane, Comment. Math. Helv. 27 (1953), 198-232.

[Sm] L. Smith, The cohomology of stable two stage Postnikov systems, Illinois J. Math. 11 (1967), 177-348.

[U] Y. Umeda, A remark on a theorem of J.-P. Serre, Proc. Japan Acad. 35 (1959), 563-566. 
POSTNIKOV TOWERS WITH FIBERS GENERALIZED EILENBERG-MAC LANE SPACES 157

[Y] D. Yau, Clapp-Puppe type Lusternik-Schnirelmann (co)category in a model category, Journal of the Korean Math. Society 39 (2002) 163-191.

[Z] A. Zabrodsky, On phantom maps and a theorem of H. Miller, Israel J. Math. 58 (1987), 129-143.

Kouyemon Iriye kiriye@mi.s.osakafu-u.ac.jp

Department of Mathematics and Information Sciences, Osaka Prefecture University, Sakai, 599-8531, Japan

Daisuke Kishimoto kishi@math.kyoto-u.ac.jp

Department of Mathematics, Kyoto University, Kyoto, 606-8502, Japan 